\title{
Effectiveness of discontinuing antibiotic treatment after three days versus eight days in mild to moderate-severe community acquired pneumonia: randomised, double blind study
}

Rachida el Moussaoui, Corianne A J M de Borgie, Peterhans van den Broek, Willem N Hustinx, Paul Bresser, Guido E L van den Berk, Jan-Werner Poley, Bob van den Berg, Frans H Krouwels, Marc J M Bonten, Carla Weenink, Patrick M

M Bossuyt, Peter Speelman, Brent C Opmeer, Jan M Prins

\begin{abstract}
Objective To compare the effectiveness of discontinuing treatment with amoxicillin after three days or eight days in adults admitted to hospital with mild to moderate-severe community acquired pneumonia who substantially improved after an initial three days' treatment.

Design Randomised, double blind, placebo controlled non-inferiority trial.

Setting Nine secondary and tertiary care hospitals in the Netherlands.

Participants Adults with mild to moderate-severe community acquired pneumonia (pneumonia severity index score $\leq 110$ ). Interventions Patients who had substantially improved after three days' treatment with intravenous amoxicillin were randomly assigned to oral amoxicillin $(n=63)$ or placebo $(\mathrm{n}=56)$ three times daily for five days.

Main outcome measures The primary outcome measure was the clinical success rate at day 10. Secondary outcome measures were the clinical success rate at day 28 , symptom resolution, radiological success rates at days 10 and 28, and adverse events. Results Baseline characteristics were comparable, with the exception of symptom severity, which was worse in the three day treatment group. In the three day and eight day treatment groups the clinical success rate at day 10 was $93 \%$ for both (difference $0.1 \%, 95 \%$ confidence interval $-9 \%$ to $10 \%$ ) and at day 28 was $90 \%$ compared with $88 \%$ (difference $2.0 \%,-9 \%$ to $15 \%)$. Both groups had similar resolution of symptoms.

Radiological success rates were $86 \%$ compared with $83 \%$ at day 10 (difference $3 \%,-10 \%$ to $16 \%$ ) and $86 \%$ compared with $79 \%$ at day 28 (difference $6 \%,-7 \%$ to $20 \%$ ). Six patients $(11 \%)$ in the placebo group and 13 patients $(21 \%)$ in the active treatment group reported adverse events $(\mathrm{P}=0.1)$.

Conclusions Discontinuing amoxicillin treatment after three days is not inferior to discontinuing it after eight days in adults admitted to hospital with mild to moderate-severe community acquired pneumonia who substantially improved after an initial three days' treatment.
\end{abstract}

\section{Introduction}

In recent guidelines for the treatment of community acquired pneumonia many recommendations could be supported by sound clinical evidence..$^{1-3}$ The duration of antibiotic therapy is, however, an exception. The usual treatment recommendation of
7-10 days for uncomplicated pneumonia is not based on scientific evidence but has nevertheless gained acceptance over the years. Two older studies in adults have suggested that a significantly shorter duration than 7-10 days might be justified. ${ }^{45}$ These studies do not, however, meet the required standards of clinical trials.

If a shorter duration of therapy is equally effective, this can be of major importance in decreasing antibiotic consumption. On a population level there is a clear relation between total antibiotic consumption and resistance rates of the pathogens. ${ }^{6-8}$ Prolonged or repeated courses of antibiotics provide the selective pressure that favours the emergence of resistant isolates. Resistance rates among common respiratory pathogens for several antimicrobial agents are increasing. ${ }^{9}$ Decreasing the duration of antibiotic treatment in respiratory tract infection might therefore decrease resistance rates. A five day, high dose course of amoxicillin for respiratory tract infections in children resulted in a significantly lower rate of carriage of penicillin resistant Streptococcus pneumoniae than the standard duration of treatment. ${ }^{10}{ }^{11}$

Two recent studies in children with non-severe pneumonia showed that three days' treatment with oral amoxicillin was as clinically effective as five days' treatment. ${ }^{12}{ }^{13}$ We carried out a randomised, double blind, placebo controlled, multicentre trial in adults admitted to hospital with mild to moderate-severe community acquired pneumonia to find out whether discontinuing treatment with amoxicillin after three days in patients showing substantial improvement is as effective as conventional treatment for eight days.

\section{Participants and methods}

The study was carried out in nine hospitals in the Netherlands between November 2000 and July 2003. Eligible patients were aged 18 years or more and had presented with clinical signs of pneumonia, a body temperature greater than $38^{\circ} \mathrm{C}$, radiological evidence of a new infiltrate consistent with pneumonia, and a pneumonia severity index score of 110 or less. ${ }^{14}$ This index stratifies patients according to risk of death within 30 days. We also considered patients aged 65 years or more with a temperature below $38^{\circ} \mathrm{C}$ as eligible if they had evident clinical signs of pneumonia and abnormalities on a chest radiograph.

Members of the study group are on bmj.com 


\section{Research}

We excluded pregnant women and patients with a history of allergy to amoxicillin; neutropenia $\left(<1.0 \times 10^{9} / \mathrm{l}\right)$; HIV infection with an indication for prophylaxis against pneumocystis pneumonia; agammaglobulinaemia; asplenia; life expectancy less than one month; treatment with an effective antimicrobial agent for more than 24 hours before admission; any other infection necessitating treatment with systemic antibiotics; recent admittance to a hospital or nursing home; serious respiratory insufficiency (arterial partial pressure of oxygen $<6.67 \mathrm{kPa}$ ); admittance to an intensive care unit; empyema; and suspicion of aspiration, atypical, Klebsiella, or staphylococcal pneumonia.

\section{Study design}

We treated patients, who had given written informed consent, with intravenous amoxicillin, the preferred empirical treatment in the Netherlands for mild to moderate-severe community acquired pneumonia. ${ }^{15}$ After 72 hours of treatment we rated four respiratory symptoms (dyspnoea, cough, coughing up sputum, colour of sputum) and general improvement on a five point symptom scale each. For respiratory symptoms the scales ranged from -2 (worsening) to 3 (complete recovery) and for general improvement from -2 (not recovered) to 3 (completely recovered). Patients who improved by two or more points on this scale, who had a temperature less than $38^{\circ} \mathrm{C}$, and who were able to take oral drugs were randomised to $750 \mathrm{mg}$ oral amoxicillin or placebo three times daily for five days. In case a causative pathogen was isolated, the pathogen had to be susceptible to amoxicillin.

The randomisation scheme was generated centrally in site specific blocks of four, with two sets of amoxicillin and two of placebo in each block. Allocation of the study drug was double blind. An independent safety monitoring committee evaluated all treatment failures after 50 patients had been randomised, and after 100 randomisations. Their first evaluation resulted in the additional exclusion criterion of a substantial amount of pleural fluid on chest radiography.

\section{Assessments}

We evaluated eligible patients at enrolment into the study and after three days of treatment. Randomised patients were further evaluated at days 7, 10 (two days after treatment ended), 14, and 28.

At baseline we obtained a complete medical history and a sample of sputum for culture. We scored pneumonia related symptoms (30 days before admission and at enrolment) using the community acquired pneumonia score, which is based on a short questionnaire. This validated questionnaire contains eight items for adults admitted to hospital with mild to moderatesevere community acquired pneumonia, ${ }^{16}$ based on respiratory symptoms and on wellbeing. Low values indicate more severe symptoms.

At each follow-up we repeated the evaluation of community acquired pneumonia score, a physical examination including vital signs, laboratory analyses, and sputum cultures. At baseline and day 28 we collected serum for determination of antibodies against respiratory viruses, Mycoplasma pneumoniae, Legionella pneumophila, and Chlamydia pneumoniae. Chest radiography was carried out at baseline and at day 10. In case of persistence of chest abnormalities, radiography was repeated at day 28. Adverse events were recorded up to day 10.

\section{Outcome measures}

We assessed study outcomes at day 10, indicated as test of cure, and at late follow-up (day 28). Our primary outcome measure was clinical cure rate at test of cure. Secondary outcome measures were clinical success rates at late follow-up, severity of symptoms, bacteriological and radiological success rates, all at test of cure and late follow-up, length of hospital stay, and adverse events.

We assessed clinical cure using established definitions, with some small adjustments (box). ${ }^{17}$ All cases not meeting the criteria for cure were classified by an independent clinical review committee as either treatment failures or indeterminate using a majority rule, before unblinding of study treatment. Symptom resolution was assessed using the community acquired pneumonia score. ${ }^{16}$ Bacteriological outcome was assessed at test of cure and late follow-up using established definitions. ${ }^{17}$ Radiological outcome was assessed at test of cure and late follow-up (see box for definitions).

\section{Statistical analysis}

The study was designed to exclude inferiority of early discontinuation of amoxicillin treatment, defined with a $10 \%$ margin in the clinical success rate. Following established guidelines ${ }^{17}$ we evaluated clinical outcome in a per protocol analysis, including all randomised patients who had been given the study drug for a minimum of 24 hours (in case of treatment failure) or who had taken at least $80 \%$ of the study drug (in case of clinical cure), and who completed follow-up ${ }^{17}$ (that is, excluding indeterminate cases).

\section{Definitions of clinical and radiological responses ${ }^{17}$}

\section{Clinical response}

\section{Test of cure}

Cure-resolution or improvement of symptoms and clinical signs related to pneumonia without the need for additional or alternative antibiotic therapy

Failure--persistence or progression of all signs and symptoms of the acute process after randomisation or the development of a new pulmonary or extrapulmonary respiratory tract infection, or the progression of abnormalities on chest radiograph after randomisation, or death due to pneumonia, or the inability to complete the study owing to adverse events

Indeterminate-patient receives less than $80 \%$ of the study drug for reasons other than clinical failure, a concomitant infection outside the respiratory tract requiring antibiotic treatment, lost to follow-up, or death unrelated to the primary diagnosis

Late follow-up

Cure-continued resolution or improvement of symptoms and clinical signs related to pneumonia without the need for additional or alternative antibiotic therapy

Recurrence-new or worsened signs and symptoms of pneumonia or another respiratory tract infection in a patient who was cured at the visit for test of cure

Indeterminate-lost to follow-up, a concomitant infection outside the respiratory tract requiring antibiotic treatment, or death unrelated to the primary diagnosis

\section{Radiological response}

Resolved

Areas of consolidation completely resolved

Improved

Areas of consolidation still exist but show evidence of clearing

Unchanged or worse

Areas of consolidation remain unchanged or show increased density 
In addition, we included in an intention to treat analysis all randomised patients who received at least one dose of the study drug. In this analysis we considered indeterminate cases as treatment failures. We carried forward all treatment failures and indeterminate cases at test of cure in the analyses at late follow-up.

We analysed bacteriological outcome only in patients with an isolated pathogen at entry to the study. At test of cure and late follow-up we defined bacteriological success as demonstrated eradication or presumed eradication (in patients who were clinically cured), and bacteriological failure as persistence ${ }^{17}$ or presumed persistence (in patients who were considered clinical failures). All other cases were considered indeterminate for analyses. We defined radiological outcome as success in patients with resolved or improved chest abnormalities and as failure with persistent or worsened chest abnormalities. ${ }^{17}$ We considered patients without repeated chest radiography at test of cure or end of follow-up (in case of persistence of chest abnormalities at test of cure) as indeterminate at those assessments.

We calculated differences in success rates, with 95\% confidence intervals. Bootstrap confidence intervals were used to estimate the mean length of hospital stay and the difference between the two groups.

\section{Results}

A total of 186 patients were enrolled in the study (fig 1). Between enrolment and randomisation (day 3) 19 patients withdrew their consent for participation, 41 did not meet the prespecified criteria for randomisation, and five were not randomised for other reasons. In total, 121 patients were randomised, of whom two were subsequently excluded because of protocol violations, leaving 119 patients.

The treatment groups had similar baseline characteristics, except for number of smokers and symptoms at admission, which were more severe in the three day treatment group (table 1 and fig 2). Pathogens were detected in 64 of the 119 (54\%) patients. Pneumonia was caused by a single organism in $84 \%$ : $92 \%$ in the three day treatment group and $75 \%$ in the eight day treatment group.

The 46 patients who did not qualify for randomisation after three days of treatment were similar to the randomised patients for all personal and baseline characteristics (data not shown). The contribution of Escherichia coli, Staphylococcus aureus, and Pseudomonas aeruginosa, however, was higher in the nonrandomised group (20\% v 1.7\%). During follow-up, antibiotic treatment was more often switched in the non-randomised group $(37 \%$ v 11\%), parapneumonic effusions occurred more often $(11 \% v 2.4 \%)$, and the average length of hospital stay was significantly longer ( 11 days $v 6$ days; $\mathrm{P}<0.01$ ).

\section{Primary outcome measures}

Table 2 shows the clinical success rates at test of cure. Overall, four treatment failures occurred in each group. In the per protocol analysis the cure rates were $93 \%(50 / 54)$ in the three day treatment group compared with $93 \%(56 / 60)$ in the eight day treatment group (difference $0.1 \%,-9 \%$ to $10 \%$ ). In the intention to treat analysis 50 of 56 patients $(89 \%)$ in the three day treatment group and 56 of $63(89 \%)$ in the eight day treatment group were considered cured (difference $0.4 \%,-11 \%$ to $12 \%$ ).

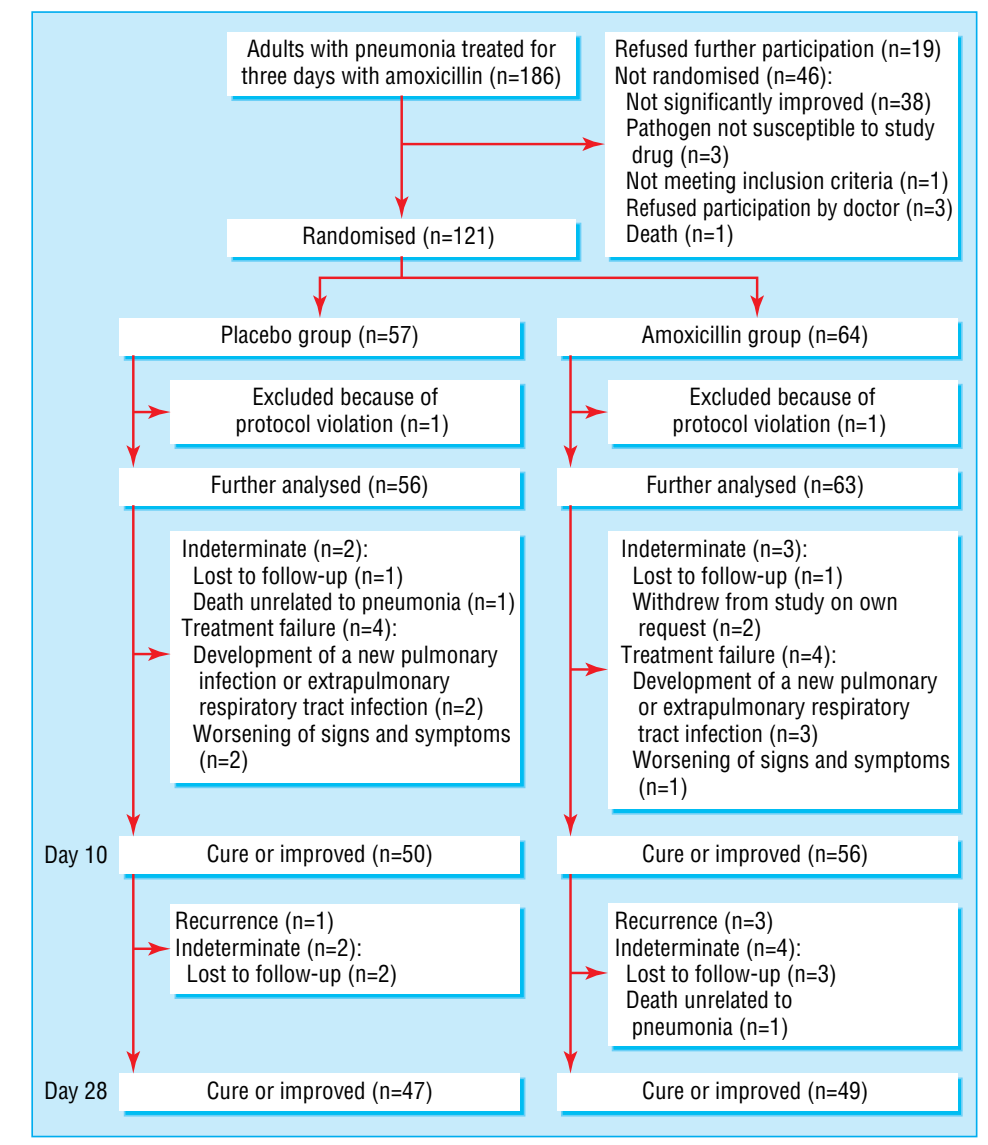

Fig 1 Trial profile 


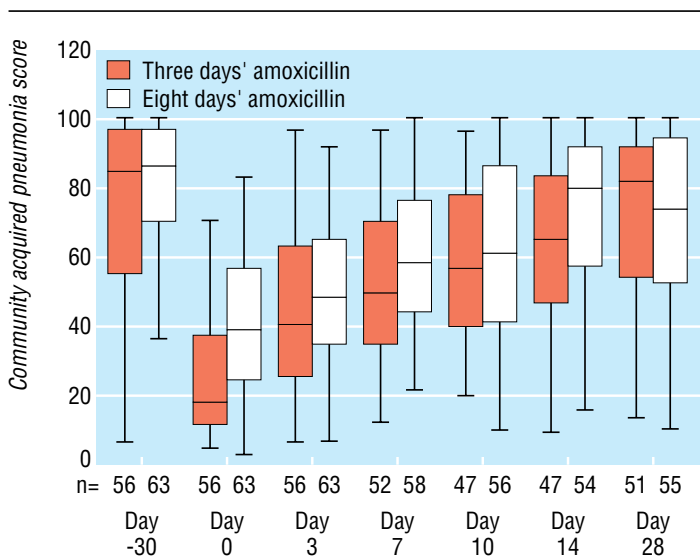

Fig 2 Community acquired pneumonia scores (medians, interquartile ranges, 10th to 90th centiles) during treatment and follow-up. Day $-30=s c o r e$ before pneumonia; day $0=$ start of treatment; day $10=$ test of cure; day $28=$ end of follow-up

\section{Secondary outcomes}

At end of follow-up the cure rates in the per protocol analysis were $90 \%(47 / 52)$ in the three day treatment group compared

Table 1 Baseline characteristics of adults with community acquired pneumonia randomised to oral placebo or oral amoxicillin for five days after three days' amoxicillin treatment. Values are numbers (percentages) unless stated otherwise

\begin{tabular}{|c|c|c|}
\hline Characteristic & $\begin{array}{l}\text { Three day treatment } \\
\text { group }(n=56)\end{array}$ & $\begin{array}{l}\text { Eight day treatment } \\
\text { group }(n=63)\end{array}$ \\
\hline$\overline{M e n}$ & $34(61)$ & 37 (59) \\
\hline Women & $22(39)$ & $26(41)$ \\
\hline Median (interquartile range) age (years) & $54(40-70)$ & $60(40-74)$ \\
\hline \multicolumn{3}{|l|}{ Conditions: } \\
\hline Underlying disease $^{*}$ & $39(70)$ & $40(64)$ \\
\hline Chronic obstructive pulmonary disease & $14(25)$ & $16(25)$ \\
\hline Frequent pneumonia $\dagger$ & $8(14)$ & $11(18)$ \\
\hline Other lung disease & $6(11)$ & $6(10)$ \\
\hline Diabetes mellitus & $9(16)$ & $7(11)$ \\
\hline Cardiovascular disease & $11(20)$ & $13(21)$ \\
\hline Smoker & $31(55)$ & $17(27)$ \\
\hline \multicolumn{3}{|l|}{ Pneumonia severity index score: } \\
\hline I & $7(13)$ & $11(18)$ \\
\hline II & $26(46)$ & $26(41)$ \\
\hline III & $17(30)$ & $17(27)$ \\
\hline IV & $6(11)$ & $9(14)$ \\
\hline $\begin{array}{l}\text { Median (interquartile range) community } \\
\text { acquired pneumonia score }\end{array}$ & $18(11-39)$ & $39(24-57)$ \\
\hline Mean $(\mathrm{SD})$ temperature $\left({ }^{\circ} \mathrm{C}\right)$ & $38^{\circ} 8(0.9)$ & $38^{\circ} 8(1.0)$ \\
\hline Mean (SD) white cell count $\left(\times 10^{9} / \mathrm{l}\right)$ & $17^{\circ} 7(7.6)$ & $15^{\circ} 5(5.2)$ \\
\hline \multicolumn{3}{|l|}{ Radiological findings: } \\
\hline Unilateral infiltrate & $51(91)$ & $56(89)$ \\
\hline Bilateral & $5(9)$ & $7(11)$ \\
\hline Single lobe & $47(84)$ & $52(83)$ \\
\hline Multiple lobe & $9(16)$ & $11(18)$ \\
\hline Pleural effusion & $7(13)$ & $2\left(3^{\circ} 2\right)$ \\
\hline Detected pathogen at study entry: & $33(59)$ & $31(49)$ \\
\hline Streptococcus pneumoniae & $19(6) \ddagger$ & $18(8) \ddagger$ \\
\hline Haemophilus influenzae & 6 & 4 \\
\hline Moraxella catharrhalis & 1 & 3 \\
\hline Haemophilus parainfluenzae & 0 & 1 \\
\hline Influenza $\mathrm{A}$ or $\mathrm{B}$ & 2 & 2 \\
\hline Chlamydia pneumoniae & 1 & 1 \\
\hline Mycoplasma pneumoniae & 0 & 0 \\
\hline Legionella pneumophila & 0 & 0 \\
\hline Other & 4 & 2 \\
\hline
\end{tabular}

* Some patients had more than one.

tMore than one episode a year.

†Number with positive blood culture result in brackets. with $88 \%(49 / 56)$ in the eight day treatment group (difference $2 \%,-9 \%$ to $15 \%)$. In the intention to treat analysis the cure rates were $84 \%$ in the three day treatment group and $78 \%$ in the eight day treatment group (difference $6 \%,-8 \%$ to $20 \%$ ). In the three day treatment group all treatment failures or recurrences were observed before the 11th day, whereas in the eight day treatment group they continued to occur until the end of follow-up (fig 3). One of the five patients who was a treatment failure or had a recurrence in the three day treatment group presented initially with confirmed pneumococcal pneumonia (S pneumoniae identified in sputum or blood culture) compared with five of seven patients in the eight day treatment group.

At test of cure a community acquired pneumonia score was available for 47 of the 56 patients in the three day treatment group and for 56 of 63 patients in the eight day treatment group (fig 2). The median score was 57 in the three day treatment group and 62 in the eight day treatment group. The baseline adjusted score at test of cure was 0.5 points ( -9 to 9 points) in favour of the three day treatment regimen.

At late follow-up the community acquired pneumonia score was available for 51 of the 56 patients in the three day treatment group and for 55 of the 63 patients in the eight day treatment group (fig 2). The median score was 82 in the three day treatment group and 74 in the eight day treatment group. Table 2 shows the bacteriological and radiological outcomes. The bootstrap estimated mean length of hospital stay was 7.9 days

Table 2 Clinical, bacteriological, and radiological outcomes for adults with community acquired pneumonia randomised to oral placebo or oral amoxicillin for five days after three days' amoxicillin treatment. Values are numbers (percentages) unless stated otherwise

\begin{tabular}{|c|c|c|c|}
\hline Outcomes & $\begin{array}{l}\text { Three day } \\
\text { treatment } \\
\text { group }\end{array}$ & $\begin{array}{l}\text { Eight day } \\
\text { treatment } \\
\text { group }\end{array}$ & Difference $(95 \% \mathrm{Cl})$ \\
\hline \multicolumn{4}{|l|}{ Day 10: } \\
\hline $\begin{array}{l}\text { Clinical cure (per protocol } \\
\text { analysis) }\end{array}$ & $50 / 54(93)$ & $56 / 60(93)$ & 0.1 ( -9 to 10$)$ \\
\hline Clinical cure & $50 / 56 \quad(89)$ & $56 / 63(89)$ & 0.4 (-11 to 12$)$ \\
\hline Bacteriological success & $22 / 25 \quad(88)$ & $19 / 20(95)$ & -7 (-23 to 9$)$ \\
\hline Radiological success & $48 / 56$ (86) & $52 / 63(83)$ & $3(-10$ to 16$)$ \\
\hline \multicolumn{4}{|l|}{ Day 28: } \\
\hline $\begin{array}{l}\text { Clinical cure (per protocol } \\
\text { analysis) }\end{array}$ & $47 / 52(90)$ & $49 / 56(88)$ & $2(-9$ to 15$)$ \\
\hline Clinical cure & $47 / 56(84)$ & 49/63 (78) & 6 (-8 to 20$)$ \\
\hline Bacteriological success & $20 / 25(80)$ & $15 / 20(75)$ & $5(-20$ to 30$)$ \\
\hline Radiological success & $48 / 56(86)$ & $50 / 63(79)$ & 6 (-7 to 20) \\
\hline
\end{tabular}

All analyses were by intention to treat, unless indicated otherwise. 


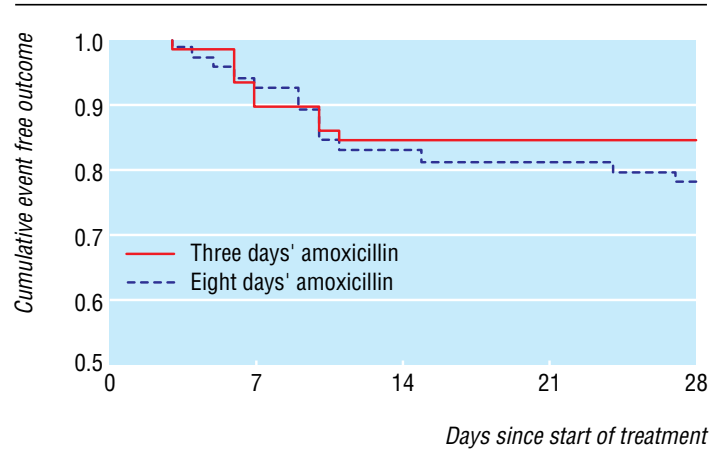

Fig 3 Proportion of patients considered clinical successes in intention to treat population. Day $3=$ day of randomisation

(6.5 to 9.3 days) in the three day treatment group compared with 8.9 days (6.8 to 11 days) in the eight day treatment group, with a bootstrap estimated mean difference of 1.0 day ( -1.3 to 3.2 days). Six patients $(11 \%)$ in the three day treatment group compared with 13 patients $(21 \%)$ in the eight day treatment group $\left(\mathrm{P}=0.1, \chi^{2}\right.$ test $)$ reported mild adverse events during or at the end of treatment. Allergy was reported only in the eight day treatment group $(\mathrm{n}=3)$.

\section{Discussion}

Discontinuing amoxicillin treatment after three days is not inferior to discontinuing it after eight days in adults admitted to hospital with mild to moderate-severe community acquired pneumonia who show substantial improvement after an initial three days' treatment. We could exclude a substantial difference in clinical success rate with a shorter treatment duration at test of cure in the per protocol analysis and at late follow-up in both the per protocol and the intention to treat analyses. In patients with (bacteraemic) pneumococcal pneumonia, treatment failure did not occur more often with short treatment duration. Both groups were also similar for symptom resolution (community acquired pneumonia scores), radiological outcomes, and mean length of hospital stay.

Our study has several potential limitations. At baseline we observed more severe symptoms (low community acquired pneumonia score) and a higher percentage of smokers in the three day treatment group. Yet we found no significant differences in the primary and secondary outcomes with the three day treatment.

In our study design we followed the Chow criteria, ${ }^{17}$ which state that the primary outcome measure should be evaluated three days after the end of treatment. This implies that recurrences in the eight day treatment group were not included as treatment failures at test of cure, which puts the three day treatment group to disadvantage.

Our results apply only to patients with mild to moderatesevere community acquired pneumonia who substantially improved after three days' amoxicillin treatment. The conclusions do not apply to the population with severe community acquired pneumonia - that is, those with a pneumonia severity index score of more than 110 or serious respiratory insufficiency. We also excluded patients with a severe immunodeficiency syndrome or a significant amount of pleural fluid. Yet of all patients with community acquired pneumonia evaluated in hospital, $60 \%$ to $80 \%$ have a pneumonia severity index score below 110 , and so do most of the patients who are not admitted to hospital with pneumonia ${ }^{14}$ including those seen by general practitioners. Our

\section{What is already known on this topic}

Recommended duration of antibiotic treatment in adults with community acquired pneumonia is not supported by scientific evidence

Three days' treatment with amoxicillin is as effective as the standard longer course in children with non-severe pneumonia

\section{What this study adds}

Discontinuing amoxicillin treatment after three days is not inferior to discontinuing it after eight days in adults with mild to moderate-severe community acquired pneumonia who have substantially improved after an initial three days' treatment

A shorter duration of treatment can help contain the growing resistance rates among respiratory pathogens

study group is comparable to that of other studies of community acquired pneumonia for most baseline characteristics such as sex, age, smoking, isolated microorganisms, the extent and distribution of chest abnormalities, ${ }^{18-23}$ and the distribution of patients among the classes I-IV on the pneumonia severity index score.

Finally, although the outcomes were comparable in both treatment groups in our study, our sample size was moderate and the possibility of chance findings cannot be fully excluded. Additional trials will have to confirm the non-inferiority of a shorter duration of treatment in adults with mild to moderate-severe community acquired pneumonia who have substantially improved after three days of treatment.

Two recent studies have convincingly shown similar clinical effectiveness with three days' oral amoxicillin treatment in children with non-severe pneumonia to that of five days' treatment. ${ }^{12}{ }^{13}$ The treatment failure rate in these studies was around 15\%-20\%, suggesting that childhood pneumonia is not a more benign condition than that of the patients we studied. Studies that have assessed the optimal duration of treatment in adults with community acquired pneumonia are rare. Two studies published in 1970 and 1983 showed that short course treatment in adults with moderately severe pneumonia was as effective as a traditional treatment for 5-6 days. ${ }^{45}$ As these studies were not carried out according to the currently required quality standards of clinical trials, their results have not been implemented in clinical practice. The effectiveness of 3-5 days of azithromycin is equivalent to that of longer treatment with a control agent. ${ }^{24-26}$ As azithromycin has a prolonged half-life, these data cannot be extended to other antibiotics. One other study compared $750 \mathrm{mg}$ levofloxacin daily for five days with 500 mg daily for 10 days in the treatment of mild to severe community acquired pneumonia. ${ }^{18}$ No statistically significant differences were found between the treatment durations.

Antibiotics used for infections of the upper and lower respiratory tract account for $75 \%$ of prescriptions in the community. Community acquired pneumonia is also one of the most important indications for antibiotic prescriptions in hospitals. A decrease in treatment duration may therefore have a significant effect on overall antibiotic consumption. As antibiotic consumption is the most important determinant of antimicrobial resistance ${ }^{6-8}$ a shorter duration of treatment can help contain the growing resistance rates among respiratory pathogens. ${ }^{9}$ 


\section{Research}

We thank GlaxoSmithKline for providing the amoxicillin and placebo capsules; the patients and investigators who participated in this trial; the medical and nursing staff of the participating centres; and M F Peeters, Tilburg, for determination of antibodies against Legionella pneumophila.

Contributors: ReM coordinated the execution of the study, supervised patients' enrolment and follow-up, analysed and interpreted the data, and wrote the first draft of the paper. JMP, PS, and PMMB designed the study protocol and supervised patients' enrolment and follow-up, interpreted the data, and prepared the paper. JMP is guarantor. CAJMdB and BCO supervised the statistical analysis, interpreted the data, and prepared the paper. PvdB contributed to the study design and coordination. PvdB, WNH, PB, GELvdB, J-WP, BvdB, FHK, MJMB, and CW contributed to patients' enrolment and follow-up and the final version of the manuscript.

Funding: Healthcare insurance board, Amstelveen, Netherlands (grant OG99-038).

Competing interests: None declared.

Ethical approval: This study was approved by the ethics committees of the participating institutions.

1 BTS guidelines for the management of community acquired pneumonia in adults Thorax 2001;56(suppl 4):IV1-64.

2 Bartlett JG, Dowell SF, Mandell LA, File TM Jr, Musher DM, Fine MJ. Practice guidelines for the management of community-acquired pneumonia in adults. Infectious Disease Society of America. Clin Infect Dis 2000;31:347-82.

3 Niederman MS, Mandell LA, Anzueto A, Bass JB, Broughton WA, Campbell GD, et al Guidelines for the management of adults with community-acquired pneumonia. Diagnosis, assessment of severity, antimicrobial therapy, and prevention. Am J Respir Crit nosis, assessment of severity, $2001 ; 163: 1730-54$.
Care

4 Sutton DR, Wicks AC, Davidson L. One-day treatment for lobar pneumonia. Thorax Sutton DR, Wicks

5 Ree GH, Davis M. Treatment of lobar pneumonia in Papua New Guinea: short course chemotherapy with penicillin or chloramphenicol. J Infect 1983;6:29-32.

6 Seppala H, Klaukka T, Vuopio-Varkila J, Muotiala A, Helenius H, Lager K, et al. The effect of changes in the consumption of macrolide antibiotics on erythromycin resistance in group A streptococci in Finland. Finnish Study Group for Antimicrobial Resistance. N Engl J Med 1997;337:441-6.

7 Bronzwaer SL, Cars O, Buchholz U, Molstad S, Goettsch W, Veldhuijzen IK, et al. A European study on the relationship between antimicrobial use and antimicrobial resistance. Emerg Infect Dis 2002;8:278-82.

8 Goossens H, Ferech M, Vander SR, Elseviers M. Outpatient antibiotic use in Europe and association with resistance: a cross-national database study Lancet 2005:365:579and 87.

9 Felmingham D, Gruneberg RN. The Alexander project 1996-1997: latest susceptibility data from this international study of bacterial pathogens from community-acquired lower respiratory tract infections. J Antimicrob Chemother 2000;45:191-203.

10 Schrag SJ, Pena C, Fernandez J, Sanchez J, Gomez V, Perez E, et al. Effect of short-course, high-dose amoxicillin therapy on resistant pneumococcal carriage: a randomized trial. JAMA 2001;286:49-56.

11 Guillemot D, Carbon C, Balkau B, Geslin P, Lecoeur H, Vauzelle-Kervroedan F, et al Low dosage and long treatment duration of beta-lactam: risk factors for carriage of penicillin-resistant Streptococcus pneumoniae. JAMA 1998;279:365-70.

12 Pakistan Multicentre Amoxycillin Short Course Therapy (MASCOT) pneumonia study gakistan Multicentre Amoxycillin Short Course Therapy (MASCOA efficacy of 3 days versus 5 days of oral amoxicillin for treatment of
group. group. Clinical efficacy of 3 days versus 5 days of oral amoxicillin for treatment
childhood pneumonia: a multicentre double-blind trial. Lancet 2002;360:835-41.

13 Agarwal G, Awasthi S, Kabra SK, Kaul A, Singhi S, Walter SD. Three day versus five day treatment with amoxicillin for non-severe pneumonia in young children: a multicentre randomised controlled trial. BMJ 2004;328:791-4.

14 Fine MJ, Auble TE, Yealy DM, Hanusa BH, Weissfeld LA, Singer DE, et al. A prediction rule to identify low-risk patients with community-acquired pneumonia. $N$ Engl J Med 1997;336:243-50.

15 Schouten JA, Prins JM, Bonten MJ, Degener J, Janknegt RE, Hollander JMR, et al. Revised SWAB guidelines for antimicrobial therapy of community-acquired pneumonia. Neth J Med 2005;63:323-35.

16 El Moussaoui R, Opmeer BC, Bossuyt PM, Speelman P, de Borgie CA, Prins JM. Development and validation of a short questionnaire in community acquired pneumonia. Thorax 2004;59:591-5.

17 Chow AW, Hall CB, Klein JO, Kammer RB, Meyer RD, Remington JS. Evaluation of new anti-infective drugs for the treatment of respiratory tract infections. Infectious Diseases Society of America and the Food and Drug Administration. Clin Infect Dis 1992;15(suppl 1):S62-88.

18 Dunbar LM, Wunderink RG, Habib MP, Smith LG, Tennenberg AM, Khashab MM, et al. High-dose, short-course levofloxacin for community-acquired pneumonia: a new treatment paradigm. Clin Infect Dis 2003;37:752-60.
19 Finch R, Schurmann D, Collins O, Kubin R, McGivern J, Bobbaers H, et al Randomized controlled trial of sequential intravenous (i.v.) and oral moxifloxacin compared with sequential i.v. and oral co-amoxiclav with or without clarithromycin in patients with community-acquired pneumonia requiring initial parenteral treatment. Antimicrob Agents Chemother 2002;46:1746-54.

20 Hagberg L, Torres A, van Rensburg D, Leroy B, Rangaraju M, Ruuth E. Efficacy and tolerability of once-daily telithromycin compared with high-dose amoxicillin for treatment of community-acquired pneumonia. Infection 2002;30:378-86.

21 Ortiz-Ruiz G, Caballero-Lopez J, Friedland IR, Woods GL, Carides A. A study evaluating the efficacy, safety, and tolerability of ertapenem versus ceftriaxone for the treatment of community-acquired pneunin in adults. Clin Infect Dis $2002,94: 1076$ 83.

22 Petitpretz P, Arvis P, Marel M, Moita J, Urueta J. Oral moxifloxacin vs high-dosage amoxicillin in the treatment of mild-to-moderate, community-acquired, suspected pneumococcal pneumonia in adults. Chest 2001;119:185-95.

23 Vetter N, Cambronero-Hernandez E, Rohlf J, Simon S, Carides A, Oliveria T, et al. A prospective, randomized, double-blind multicenter comparison of parenteral ertapenem and ceftriaxone for the treatment of hospitalized adults with community-acquired pneumonia. Clin Ther 2002;24:1770-85.

24 Bohte R, van't Wout JW, Lobatto S, Blusse van Oud AA, Boekhout M, Nauta EH, et al. Efficacy and safety of azithromycin versus benzylpenicillin or erythromycin in community-acquired pneumonia. Eur J Clin Microbiol Infect Dis 1995;14:182-7.

25 Hoepelman IM, Mollers MJ, van Schie MH, Greefhorst AP, Schlosser NJ, Sinninghe Damste EJ, et al. A short (3-day) course of azithromycin tablets versus a 10-day course of amoxycillin-clavulanic acid (co-amoxiclav) in the treatment of adults with lower resof amoxycillin-clavulanic acid (co-amoxiclav) in the treatment of adults with lower res-
piratory tract infections and effects on long-term outcome. Int J Antimicrob Agents piratory tract

26 O'Doherty B, Muller O. Randomized, multicentre study of the efficacy and tolerance of azithromycin versus clarithromycin in the treatment of adults with mild to moderate community-acquired pneumonia. Azithromycin Study Group. Eur J Clin Microbiol Infect Dis 1998;17:828-33.

27 Carbon C, Bax RP. Regulating the use of antibiotics in the community. $B M J$ 1998;317:663-5

(Accepted 16 March 2006)

bmj.com 2006;332:1355

Department of Internal Medicine, Division of Infectious Diseases, Tropical Department of Internal Medicine, Division of Infectious Diseases, Tropica
Medicine and AIDS, Academic Medical Center, Meibergdreef 9, $1105 \mathrm{AZ}$ Medicine and AIDS, Aca

Rachida el Moussaoui clinical research fellow

Peter Speelman professor of infectious diseases

Jan M Prins internist in infectious diseases

Department of Clinical Epidemiology and Biostatistics, Academic Medical Center, Amsterdam

Corianne A J M de Borgie clinical epidemiologist

Patrick M M Bossuyt professor of clinical epidemiology

Brent C Opmeer clinical epidemiologist

Department of Internal Medicine, Leiden University Medical Center, Leiden,

Netherlands

Peterhans van den Broek professor of infectious diseases

Department of Internal Medicine, Diaconessenhuis, Utrecht, Netherlands

Willem N Hustinx internist in infectious diseases

Department of Pulmonology, Academic Medical Center, Amsterdam

Paul Bresser pulmonologist

Department of Internal Medicine, Onze Lieve Vrouwe Gasthuis, Amsterdam Guido E L van den Berk resident

Department of Internal Medicine, Sint Lucas-Andreas Hospital, Amsterdam Jan-Werner Poley resident

Department of Pulmonology, Sint Lucas-Andreas Hospital

Bob van den Berg pulmonologist

Department of Pulmonology, Onze Lieve Vrouwe Gasthuis

Frans H Krouwels pulmonologist

Department of Internal Medicine, University Medical Center Utrecht

Marc J M Bonten professor of infectious diseases

Department of Pulmonology, Kennemer Gasthuis, Haarlem, Netherlands

Carla Weenink pulmonologist

Correspondence to:J M Prins j.m.prins@amc.uva.nl 


\section{Commentary: What is the optimal duration of antibiotic therapy?}

John Paul

For some infections, such as Staphylococcus aureus bacteraemia or enterococcal endocarditis, clear evidence favours prolonged treatment to prevent relapse. Conversely, for some situations such as surgical prophylaxis and the treatment of gonorrhoea and of uncomplicated urinary tract infections in women, roles for short courses of antibiotics are well established. In this issue, El Moussaoui et al present evidence in favour of a total of three days' treatment for uncomplicated cases of community acquired pneumonia. ${ }^{1}$ This suggests that current guidelines recommending 7-10 days should be revised. ${ }^{2}$ In fact, the lack of evidence to support short course therapy for many common infections is surprising, and it has become accepted practice to continue treatment for days after symptoms have improved.

How can we improve our knowledge? This is an important question: antibiotics may be associated with adverse reactions, and prolonged exposure to antibiotics may encourage the development or acquisition of antibiotic resistant organisms. Also, antibiotic treatment is expensive and problems may occur with compliance. Comparative outcome studies provide a solution but require large numbers to provide the statistical power for significance. Pooling of multiple studies may be affected by inconsistencies in study design but may still yield clues-for example, a recent meta-analysis showed a five day course of cephalosporin to be superior to 10 days of penicillin for streptococcal pharyngitis. ${ }^{3}$ However, as a secure evidence base for clinical decision making, prospective multicentre trials probably provide the most definitive results.

El Moussaoui et al compared outcomes for cases of mild to moderate-severe community acquired pneumonia after treatment with antibiotics for three or eight days. ${ }^{1}$ The study involved nine hospitals in the Netherlands and was carried out as a randomised, double blind, placebo controlled non-inferiority trial. Patients who met entry criteria were treated with intravenous amoxicillin. Those who showed significant improvement after 72 hours were switched to either oral amoxicillin or placebo for five days. Clinical and radiological outcomes assessed at days 10 and 28 were not significantly different.
Not only does the study yield strong evidence in favour of short course therapy for a subset of patients with community acquired pneumonia, but also shows how centres can cooperate to tackle longstanding areas of uncertainty in clinical microbiology and infectious diseases. Many other common clinical situations would repay the efforts of comparable approaches. An example would be the treatment of vascular catheter related $S$ aureus bacteraemia. ${ }^{4}$ The literature suggests a minimum treatment period of 10-14 days, but some clinicians argue from experience that shorter periods are adequate: it would be good to know what the minimum treatment period should be, especially for methicillin resistant staphylococci and in patients whose long term prognosis is poor.

When optimising therapy for an infection, a lot of variables have to be juggled, including choice of antibiotic, the patient's immune status, the infecting agent, and the nature of the septic focus. Despite such difficulties, El Moussaoui et al show that by careful selection of case definition and comparison of standardised regimens it is possible to make medicine into more of a science.

Competing interests: None declared.

El Moussaoui R, de Bourgie CAJM, van den Broek P, Hustinx WN, Bresser P, van den Berk GEL, et al. Effectiveness of discontinuing antibiotic treatment after three days ver-
sus eight days in mild to moderate-severe community acquired pneumonia: randomised, double blind study. BMJ 2006;000-00.

2 British Thoracic Society Standards of Care Committee. BTS guidelines for the management of community acquired pneumonia in adults. Thorax 2001;56(suppl IV):1-64.

3 Casey JR, Pichichero ME. Meta-analysis of short course antibiotic treatment for Group A streptococcal tonsillopharyngitis. Paediatr Infect Dis J 2005;24:909-17.

4 Fowler VG Jr, Sanders LL, Sexton DJ, Kong L, Marr KA, Gopal AK, et al. Outcome of Staphylococcus aureus bacteremia according to compliance with recommendations of 86

(Accepted 19 May 2006)

Microbiology Department, Royal Sussex County Hospital, Brighton BN2 5BE John Paul consultant

tetrix@pavilion.co.uk 